\section{LIGHTs out for asthma}

\section{By Lauren Martz, Staff Writer}

Two years after Kyowa Hakko Kirin Co. Ltd. granted sanofi-aventis Group an exclusive license to an antibody against the tumor necrosis factor ligand LIGHT to treat irritable bowel disease, researchers at the La Jolla Institute for Allergy \& Immunology have now uncovered an even larger indication for the target-asthma.

The new findings suggest that inhibiting tumor necrosis factor ligand superfamily member 14 (TNFSF14; LIGHT; CD258) could prevent airway remodeling in asthma. ${ }^{1}$

LIGHT is expressed on immune cells and acts through tumor necrosis factor receptor superfamily member 14 (TNFRSF14; LIGHTR; HVEM) and lymphotoxin $\beta$ receptor (LTBR; LT $\beta R$ ). In 1998, a team at the La Jolla Institute for Allergy \& Immunology (LIAI) identified LIGHT and showed that it promoted intestinal inflammation. ${ }^{2,3}$

Those data caught the attention of Kyowa, which now has exclusive rights to the target and an antibody against it that the company co-developed with LIAI.

In 2009, Kyowa partnered with sanofi to develop the antibody, which is in preclinical testing to treat irritable bowel disease (IBD).

Now, a new group of researchers at LIAI led by Michael Croft has shown that LIGHT also may have a role in the airway remodeling that increases airway wall thickness and decreases lung function in asthma. Croft is a division head and professor of immune regulation at LIAI.

The paper, published in Nature Medicine, also included researchers from the University of California, San Diego, Sanford-Burnham Medical Research Institute and Heinrich Heine University of Duesseldorf.

The team developed a murine fusion protein combining the $\mathrm{Fc}$ domain of IgG and the extracellular portion of $\mathrm{Lt} \beta \mathrm{r}$ that inhibits Light's interactions with its receptors, Lt $\beta r$ and Tnfrsf14.

"LIGHT has two receptors that are expressed on several cell types that appear to be directly responsible for the features of remodeling in the lung," noted Croft. "This suggests that preventing LIGHT from binding to its receptors will dampen the activities of these cells and might specifically reduce the remodeling features or prevent them from developing."

In a mouse model of allergen-induced asthma, intraperitoneal administration of the fusion protein shortly after the onset of acute inflammation decreased features of asthmatic airway remodeling compared with injection of IgG control, including fibrosis, smooth muscle hyperplasia, airway collagen expression and $\alpha$-smooth muscle actin levels.

The team also showed that genetic knockout of Light in the mice decreased these features compared with those in wild-type mice.

Pulmonary function testing showed that the fusion protein also decreased airway hyperresponsiveness. However, inhibition of Light did not alter the levels of inflammatory eosinophils in bronchoalveolar lavage fluid, suggesting that different mechanisms control inflammation and airway remodeling during asthma.

The findings were replicated in an additional mouse model of asthma induced by intranasal challenge with ovalbumin.

\section{A LIGHT remodel}

According to Patrick Ho, director of business and technology development at LIAI, the team now needs to validate the target in additional animal models of asthma that more closely mimic the condition in humans.

"The mouse model that the researchers used is economical, but it is not a good replication of human asthma. Whether this interesting work that points to new therapeutic targets can be translated into humans remains to be determined," said Yang-Xin Fu, professor of pathology at The University of Chicago.

"This is an allergen-induced model in mice, which has not always demonstrated equal validity to asthma pathological mechanisms in humans," said Annette Hastie, associate professor in the Section of Pulmonary \& Critical Care at Wake Forest School of Medicine's Center for Genomics and Personalized Medicine Research. Hastie also noted that it is not known whether the fusion protein would be effective at treating established disease.

"The reductions in the airway fibrosis, smooth muscle hyperplasia and airway hyperresponsiveness were achieved by simultaneous delivery of the fusion protein $\mathrm{Lt} \beta \mathrm{r}$-Fc during the chronic develop-Kurt Stoeckli, sanofi-aventis Group ment period of these features in the IgG-treated mice," she said.

Hastie suggested looking for a reversal of the features when a LIGHT inhibitor is delivered after the establishment of prominent airway remodeling in mice, noting that human asthma is often diagnosed after airway remodeling has occurred.

Hans-Erik Claesson, senior scientific advisor to Orexo AB, suggested that the team should assess the effects of LIGHT inhibition in combination with anti-inflammatory asthma drugs to determine whether targeting both airway inflammation and remodeling results in better control.

Hastie made the same point. "Targeting a single component of a very complex process has often failed due to redundancy throughout the immune system," she said.

Wolfgang Jarolimek, manager of biochemical pharmacology at Pharmaxis Ltd., told SciBX that "any treatment that uses the LIGHT pathway will be an add-on therapy." He added that the study shows that inhibiting 
LIGHT does not affect the inflammation underlying asthma but will control the developing fibrosis so that the disease does not worsen.

Jarolimek added, "The data from the study are exciting and will spur further research but do not provide a developable drug." Indeed, Fu also thought that the fusion protein might not be the best compound for therapeutic development. "If you really wish to establish this method, you will need to develop an antibody," he said.

Pharmaxis markets Aridol mannitol, a lung function test that measures airway hyperresponsiveness using dry mannitol powder and an inhaler.

\section{Asthma and beyond}

Kurt Stoeckli, VP of the Immuno-Inflammation Therapeutic Strategic Unit at sanofi $\mathrm{R} \& \mathrm{D}$, said that based on the Nature Medicine paper, the pharma is evaluating the potential for anti-LIGHT antibodies to treat asthma.

"There has been a proposed role for LIGHT in respiratory indications, and for the first time we have compelling evidence derived from animal pharmacology in support of this," he said. "With regard to future therapy, I think we should take into consideration that asthma today is considered a rather heterogeneous type of disease. In fact there are multiple subtypes of severe and advanced stage asthma reported where current standard of care is clearly insufficient and the unmet medical need is particularly high."

He added, "The present publication provides a new type of evidence in support of a role of LIGHT in some forms of asthma in which macrophage-driven pathology and related airway remodeling is a dominant component. In order to prove clinical utility in human therapy, I would thus anticipate that the selection of patient cohorts that express similar clinical phenotypes and related translational signatures is likely to be in the focus for proof-of-concept studies."

Orexo's Claesson said the paper's findings also point to a potential role of LIGHT in chronic obstructive pulmonary disease (COPD).
"The disease is characterized by very pronounced airway remodeling, and we have very few drugs to treat COPD. I think immunohistochemistry analysis of LIGHT expression in human lung tissues from patients with asthma or COPD would be very interesting," he said.

Orexo and Johnson \& Johnson have therapeutics in preclinical testing to treat asthma and COPD. One of them is OX-CLI, an oral bronchodilator that inhibits leukotriene C4 synthase (LTC4S).

Ho told SciBX that LIAI has filed for patent applications covering LIGHT in asthma and that the IP is available for licensing. LIAI and Kyowa did not disclose further details about their licensing agreement regarding LIGHT and its applications in asthma.

Martz, L. SciBX 4(18); doi:10.1038/scibx.2011.501

Published online May 5, 2011

\section{REFERENCE}

1. Doherty, T.A. et al. Nat. Med.; published online April 17, 2011; doi:10.1038/nm.2356

Contact: Michael Croft, La Jolla Institute for Allergy \& Immunology, La Jolla, Calif. e-mail: mick@liai.org

2. Mauri, D.N. et al. Immunity 8, 21-30 (1998)

3. Wang, J. et al. J. Immunol. 174, 8173-8182 (2005)

\section{COMPANIES AND INSTITUTIONS MENTIONED}

Heinrich Heine University of Duesseldorf, Duesseldorf, Germany Johnson \& Johnson (NYSE:JNJ), New Brunswick, N.J.

Kyowa Hakko Kirin Co. Ltd. (Tokyo:4151), Tokyo, Japan La Jolla Institute for Allergy \& Immunology, La Jolla, Calif. Orexo AB (SSE:ORX), Uppsala, Sweden Pharmaxis Ltd. (ASX:PXS; Pink:PXSLY), Frenchs Forest, New South Wales, Australia

Sanford-Burnham Medical Research Institute, La Jolla, Calif. sanofi-aventis Group (Euronext:SAN; NYSE:SNY), Paris, France University of California, San Diego, La Jolla, Calif.

The University of Chicago, Chicago, III.

Wake Forest School of Medicine, Winston-Salem, N.C. 